\title{
Migraine predicts physical and pain symptoms among psychiatric outpatients
}

\author{
Ching-l Hung ${ }^{1}$, Chia-Yih Liu ${ }^{1}$ and Shuu-Jiun Wang ${ }^{2 *}$
}

\begin{abstract}
Background: No study has been performed to compare the impacts of migraine and major depressive episode (MDE) on depression, anxiety and somatic symptoms, and health-related quality of life (HRQoL) among psychiatric outpatients. The aim of this study was to investigate the above issue.

Methods: This study enrolled consecutive psychiatric outpatients with mood and/or anxiety disorders who undertook a first visit to a medical center. Migraine was diagnosed according to the International Classification of Headache Disorders, $2^{\text {nd }}$ edition. Three psychometric scales and the Short-Form 36 were administered. General linear models were used to estimate the difference in scores contributed by either migraine or MDE. Multiple linear regressions were employed to compare the variance of these scores explained by migraine or MDE.

Results: Among 214 enrolled participants, 35.0\% had migraine. Bipolar II disorder patients (70.0\%) had the highest percentage of migraine, followed by major depressive disorder (49.1\%) and only anxiety disorder (24.5\%). Patients with migraine had worse depression, anxiety, and somatic symptoms and lower SF-36 scores than those without. The estimated differences in the scores of physical functioning, bodily pain, and somatic symptoms contributed by migraine were not lower than those contributed by MDE. The regression model demonstrated the variance explained by migraine was significantly greater than that explained by MDE in physical and pain symptoms.
\end{abstract}

Conclusions: Migraine was common and the impact of migraine on physical and pain symptoms was greater than MDE among psychiatric outpatients. Integration of treatment strategies for migraine into psychiatric treatment plans should be considered.

Keywords: Depression, Anxiety, Headache, Pain, Quality of life, Somatization

\section{Background}

Migraine, mood disorders, and anxiety disorders are comorbid with each other and interact [1-6]. Many studies have investigated the prevalence and impact of depression and/or anxiety among patients with migraine. These studies show that comorbidity with depression and/or anxiety is common and related to a poorer quality of life, increases the suicide risk, and predicts a poorer outcome among patients with migraine [7-11].

Many other studies, on the contrary, have investigated the frequency and impact of migraine among patients with mood and/or anxiety disorders [12-20]. Most of these studies evaluated the impact of migraine on

\footnotetext{
* Correspondence: sjwang@vghtpe.gov.tw

${ }^{2}$ Neurological Institute, Taipei Veterans General Hospital and National YangMing University School of Medicine, No. 201 Shi-Pai Road, Section 2, Taipei 112, Taiwan

Full list of author information is available at the end of the article
}

psychiatric outpatients with depression or major depressive disorder (MDD) [12-16]. Comorbidity with migraine is associated with more severe depression, anxiety, and somatic symptoms, as well as a poorer health-related quality of life (HRQoL) [14-16].

Patients with depression may suffer from impaired multiple functions and have an increased social burden [21]. Major depressive episode (MDE) is one of the worst states of mood disorders and may be most able to reflect functional impairments of depression. Similarly, migraine also causes significant functional impairment in multiple dimensions [22]. To the best of our knowledge, no study has been performed to compare the impacts of migraine and MDE on depression, anxiety and somatic symptoms as well as HRQoL among psychiatric outpatients with mood and anxiety disorders (or non-psychotic outpatients).

\section{实}


Understanding the impact of migraine among psychiatric outpatients with mood and/or anxiety disorder is important because the information might alert physicians to note migraine and to integrate the treatment plan for mood disorders, anxiety, and migraine. Therefore, the study aimed to compare the differences in depression, anxiety and somatic symptoms, as well as HRQoL, associated with the presence of migraine or MDE among psychiatric outpatients with mood and/or anxiety disorders. We hypothesized that psychiatric outpatients with migraine have a greater severity of depression, anxiety, and somatic symptoms as well as a poorer HRQoL based on previous studies [12-16]. Moreover, the impacts of migraine on physical and pain symptoms might be as important as those of a current MDE.

\section{Methods}

\section{Subjects}

This study enrolled subjects between September 2007 and August 2009 in the psychiatric outpatient clinic of Chang Gung Memorial Hospital, a medical center in northern Taiwan. The study participants were consecutive psychiatric outpatients (20-60-years-old) who visited our clinic for the first time and had not taken any medications for psychiatric disorders and migraine prevention within the previous 4 weeks. Four exclusion criteria were established: 1) psychotic disorders, such as schizophrenia, delusional disorder, and other psychotic disorders; 2) mental retardation, delirium, dementia, and mental disorders due to general medical conditions; 3) psychotic symptoms, catatonic features, severe psychomotor retardation, or a current manic episode in the index month, which may cause difficulty in completing self-administered scales or cooperating with the study process; 4) a history of substance dependence or abuse without full remission in the index month.

The government-run single-payer compulsory $\mathrm{Na}$ tional Health Insurance program in Taiwan enrolls more than 96\% of Taiwan's population [23]. The insured in Taiwan have complete freedom to choose their health care providers. They can seek care at tertiary care institutions, regardless of the severity of their illness. Only $2.3 \%$ of patients in the clinics of a university teaching hospital in Taiwan were referred from primary clinics [24]. Therefore, although our patients were collected from a medical center, they did not differ much in the demographic patterns of those attending primary psychiatric clinics.

The study was approved by the Institutional Review Board of Chang Gung Memorial Hospital. A written informed consent, based on the guidelines regulated in the Declaration of Helsinki, was obtained from all subjects prior to study enrollment.

\section{Assessment of the severity of depression, anxiety, and somatic symptoms, and HRQoL}

The Hamilton Depression Rating Scale (HAMD), the Depression and Somatic Symptoms Scale (DSSS), and the Hospital Anxiety and Depression Scale (HADS) were used to evaluate depression, anxiety, and somatic symptoms over the previous week [25-29].

The DSSS is a self-administered scale composed of 12 items in the Depression Subscale (DS) and 10 items in the Somatic Subscale (SS), which includes five pain symptoms and five non-pain somatic symptoms. The reliability and validity of the DSSS have been reported in previous studies [25-27].

The HADS comprises 7 items in the anxiety subscale (HADS-A) and 7 items in the depression subscale (HADS-D) and does not include any somatic symptoms [29]. Therefore, the HADS can measure depression and anxiety without being confounded by somatic symptoms.

The three scales were used simultaneously to investigate the severity of depression, anxiety, and somatic symptoms. The total scores for each scale or subscale range from 0 to 36 for the DS and 0 to 30 for the SS, 0 to 52 for the HAMD, and 0 to 21 for the HADS-D and HADS-A. A higher score indicates a greater severity.

The acute version of the Short-Form 36 (SF-36), which evaluated the HRQoL in the past week with the same items as the SF-36, was used to be compatible with the evaluating period of the three psychometric scales. The SF-36 includes eight domains: physical functioning (PF), role limitations-physical (RP), bodily pain (BP), general health perceptions $(\mathrm{GH})$, vitality (VT), social functioning (SF), role limitations-emotional (RE), and mental health $(\mathrm{MH})$ [30]. A higher score indicates a better HRQoL. The scores of each subscale ranged from 0 (lowest wellbeing) to 100 (highest well-being). According to the SF-36 Health Survey Manual, a difference of more than 5 points in each subscale of the SF-36 is considered clinically significant. The Taiwanese version of the SF-36 shows good validity and reliability [31].

The DSSS, HADS, SF-36 were self-administered scales. The HAMD was assessed by a psychiatrist, who was blinded to the results of other psychometric scales, psychiatric diagnoses, and headache diagnoses.

\section{Diagnoses of psychiatric disorders}

Another psychiatrist, who was blinded to the diagnosis of headache and the results of these scales, used the Structured Clinical Interview for DSM-IV-TR to diagnose mood and anxiety disorders [32]. If patients had mood and anxiety disorders simultaneously, their diagnoses were categorized as bipolar disorders or MDD, not anxiety 
disorders. Therefore, this study divided patients into bipolar disorders, MDD, anxiety-only disorders, and others.

\section{Assessment of headache Headache diagnosis}

All patients completed a structured headache intake form, designed to meet the operational criteria of the International Classification of Headache Disorders, 2nd edition (ICHD-2) [33] including information for the diagnoses of migraine and other common headaches, such as headache frequency, intensity, features, aura, locations, duration, and precipitating factors, as well as the amount and frequency of use of drugs taken for pain. An experienced headache specialist (neurologist), who was blinded to the results of the psychiatric diagnoses and psychometric scales, interviewed all patients after they had completed the headache intake form and made headache diagnoses based on the ICHD-2. Patients with chronic migraine (CM) with or without medication-overuse headache $(\mathrm{MOH})$ and episodic migraine (EM) (i.e. migraine with or without aura) were categorized as the migraine group.

In order to understand the synergic effects of a current MDE and migraine on depression, anxiety, and somatic symptoms, patients were divided into four groups: a current MDE with migraine, a current MDE without migraine, migraine without a current MDE, and others (neither a current MDE nor migraine).

\section{Headache intensity and frequency assessment}

Subjects evaluated their average headache intensity during the previous week using a visual analog scale, with 0 representing "no headache" and 10 representing "headache as severe as I can imagine." The total number of headache days in the past week was also collected.

\section{Statistical methods}

We used SPSS for Windows 12.0 to perform the statistical analyses. In performing independent $t$-tests, Bonferroni corrections were used due to multiple comparisons (statistical significance: $p<0.01$ for HAMD, DS, SS, HADS-D, and HADS-A and $p<0.006$ for the eight SF-36 subscales). One-way analysis of variance (ANOVA) with post-hoc comparisons by Scheffe's method was used to test the differences in continuous variables among groups. The Chi-square and Pearson's correlation tests were used in appropriate situations.

To compare the impacts of migraine and MDE, multiple linear regression analyses with forward selection, which could prevent multicolinearity, were performed. The dependent variables were the scores of the depression, anxiety, and somatic symptoms, as well as the SF-36 subscales. All these dependent variables were continuous variables. Therefore, using multiple linear regression analyses was appropriate. The independent variables included migraine, a current MDE, and five demographic variables (age, gender, educational level, marital status, and employment). Moreover, general linear models were used to calculate the estimated difference contributed by either migraine or MDE after controlling for demographic variables. In statistical analyses, a two-tailed $p$ value $<0.05$ was considered statistically significant.

\section{Results}

\section{Subjects}

During the study period, 231 patients fulfilled the criteria for enrollment. Of them, 17 patients refused to participate and 214 (92.6\%) subjects completed the study. Table 1 demonstrates that $35.0 \%$ of these patients had migraine and $15.9 \%$ had chronic daily headache (CM with or without $\mathrm{MOH}$ or chronic tension-type headache).

Table 2 shows the percentages of migraine among psychiatric diagnoses. Patients with bipolar II disorder had the highest percentage of migraine $(70.0 \%)$, followed by MDD (49.1\%), only anxiety disorder, and no mood and/ or anxiety disorders. Table 3 shows the demographic variables and demonstrates that female patients had a significantly higher percentage of migraine. There was no significant difference in age, educational years, employment, and marital status between patients with and without migraine.

Patients with migraine was associated with a higher risk of being in a current $\operatorname{MDE}(69.3 \%$ vs. $30.9 \%$, odds ratio $(\mathrm{OR})=5.05,95 \% \mathrm{CI}=2.75-9.28, p<0.001)$ compared to those without migraine. Similarly, patients with migraine also had a higher risk of a past suicide attempt history than those without $(30.7 \%$ vs. $13.7 \%, \mathrm{OR}=2.79,95 \%$ $\mathrm{CI}=1.40-5.57, p<0.01)$.

\section{Comparisons of depression, anxiety, and somatic} severities between patients with and without migraine

Table 3 shows the severities of depression, anxiety, and somatic symptoms between patients with and without migraine and with and without a current MDE. Compared to

Table 1 The percentages of headache diagnoses

\begin{tabular}{lcc}
\hline Headache diagnosis & Number & Percentage \\
\hline Chronic migraine $^{\mathrm{a}}$ & 30 & $\mathbf{1 4 . 0}$ \\
Episodic migraine $^{\mathrm{b}}$ & 45 & $\mathbf{2 1 . 0}$ \\
Probable migraine & 29 & $\mathbf{1 3 . 6}$ \\
Chronic tension-type headache & 4 & $\mathbf{1 . 9}$ \\
Episodic tension-type headache & 40 & $\mathbf{1 8 . 7}$ \\
Probable tension-type headache & 26 & $\mathbf{1 2 . 1}$ \\
Other headache & 12 & $\mathbf{5 . 6}$ \\
No headache & 28 & $\mathbf{1 3 . 1}$ \\
Total & 214 & $\mathbf{1 0 0}$ \\
\hline
\end{tabular}

a Eight (26.7\%) patients also had medication-overuse headache.

${ }^{b}$ Six $(13.3 \%)$ of them had migraine with aura. 
Table 2 The percentage of patients with migraine by individual psychiatric diagnoses

\begin{tabular}{lcc}
\hline & Number & Percentage \\
\hline Bipolar II disorder & $7 / 10$ & $\mathbf{7 0 . 0}$ \\
Major depressive disorder & $54 / 110$ & $\mathbf{4 9 . 1}$ \\
Only anxiety disorder & $12 / 49$ & $\mathbf{2 4 . 5}$ \\
No above diagnosis & $2 / 45$ & $\mathbf{4 . 4}$ \\
Total & $75 / 214$ & $\mathbf{3 5 . 0}$ \\
\hline
\end{tabular}

patients without migraine, those with migraine had a significantly $(p<0.01)$ greater severity of depression (HAMD, HADS-D, DS), anxiety (HADS-A), and somatic symptoms (SS). Compared to patients without a current MDE, patients with a current MDE also had a significantly greater severity of depression, anxiety, and somatic symptoms. After controlling for demographic variables by a general linear model, the estimated difference in the SS score contributed by migraine was slightly greater than that contributed by a current MDE (6.2 vs. 5.3).

Figure 1 shows the scores of depression, anxiety, and somatic symptoms in four subgroups. There was a trend that patients with a current MDE and migraine had the greatest severities of depression, anxiety, and somatic symptoms, followed by patients with a current MDE without migraine, patients with migraine without a current MDE, and patients without a current MDE or migraine, with the exception of the SS.

\section{Comparisons of the SF-36 subscales between patients with and without migraine}

Table 4 shows the differences in the SF-36 subscales between patients with and without migraine. Patients with migraine had significantly $(p<0.006)$ lower scores (a poorer HRQoL) in the eight subscales as compared with patients without migraine. Similarly, patients with a current MDE also had significantly lower scores in all subscales. After controlling for demographic variables by a general linear model, the estimated differences between migraine $v s$. non-migraine and between a current MDE vs. no current MDE reached statistical significance and clinical significance (a difference in score $>5$ ) in all eight subscales. Of note, the reduction attributed to migraine was slightly greater than that attributed to a current MDE in the PF (11.2 vs. 9.4) and BP (23.5 vs. 20.0) scores.

Table 3 Comparison of depression, anxiety, and somatic symptoms as well as demographic variables between outpatients with and without migraine and MDE

\begin{tabular}{|c|c|c|c|c|c|}
\hline & Total $(n=214)$ & & Yes & No & Estimated difference $^{a}$ \\
\hline \multirow[t]{2}{*}{ HAMD } & $18.3 \pm 6.3$ & Migraine $(n=75)$ & $21.6 \pm 5.5^{* *}$ & $16.5 \pm 6.1$ & $4.7^{* *}$ \\
\hline & & $\operatorname{MDE}(n=95)$ & $23.0 \pm 4.1^{* *}$ & $14.5 \pm 5.3$ & $8.1^{* *}$ \\
\hline \multirow[t]{2}{*}{ DS } & $18.9 \pm 8.6$ & Migraine & $24.1 \pm 7.9^{* *}$ & $16.1 \pm 7.6$ & $7.3^{* *}$ \\
\hline & & $\mathrm{MDE}$ & $25.3 \pm 6.0^{* *}$ & $13.8 \pm 6.7$ & $10.9^{* *}$ \\
\hline \multirow[t]{2}{*}{ SS } & $13.0 \pm 7.0$ & Migraine & $17.5 \pm 6.7^{* *}$ & $10.5 \pm 5.9$ & $6.2^{* *}$ \\
\hline & & MDE & $16.1 \pm 7.1^{* *}$ & $10.4 \pm 5.9$ & $5.3^{* *}$ \\
\hline \multirow[t]{2}{*}{ HADS-D } & $10.3 \pm 5.0$ & Migraine & $12.8 \pm 4.5^{* *}$ & $8.9 \pm 4.7$ & $3.8^{* *}$ \\
\hline & & MDE & $13.7 \pm 3.7^{* *}$ & $7.5 \pm 4.0$ & $6.1^{* *}$ \\
\hline \multirow[t]{2}{*}{ HADS-A } & $12.1 \pm 4.4$ & Migraine & $13.9 \pm 4.0^{* *}$ & $11.2 \pm 4.4$ & $2.6^{* *}$ \\
\hline & & $\mathrm{MDE}$ & $14.4 \pm 3.5^{* *}$ & $10.3 \pm 4.3$ & $4.0^{* *}$ \\
\hline \multirow[t]{2}{*}{ Age (years) } & $38.3 \pm 10.5$ & Migraine & $38.1 \pm 9.6$ & $38.5 \pm 10.9$ & \\
\hline & & MDE & $36.3 \pm 10.0^{*}$ & $40.0 \pm 10.6$ & \\
\hline \multirow[t]{2}{*}{ Educational years } & $12.5 \pm 2.9$ & Migraine & $12.2 \pm 2.9$ & $12.7 \pm 3.0$ & \\
\hline & & MDE & $12.5 \pm 2.7$ & $12.5 \pm 3.1$ & \\
\hline \multirow[t]{2}{*}{ Gender (female, \%) } & 63.1 & Migraine & $82.7^{* *}$ & 52.5 & \\
\hline & & MDE & 67.4 & 59.7 & \\
\hline \multirow[t]{2}{*}{ Employed (yes, \%) } & 66.4 & Migraine & 60.0 & 69.8 & \\
\hline & & MDE & 65.3 & 67.2 & \\
\hline \multirow[t]{2}{*}{ Married (yes, \%) } & 56.5 & Migraine & 56.0 & 56.8 & \\
\hline & & MDE & $47.4^{*}$ & 63.9 & \\
\hline
\end{tabular}

HAMD Hamilton Depression Rating Scale, DS depression subscale of the Depression and Somatic Symptoms Scale (DSSS), SS somatic subscale of the DSSS, HADSD depression subscale of the Hospital Anxiety and Depression Scale (HADS), HADS-A anxiety subscale of the HADS, MDE a current major depressive episode. ${ }^{a}$ Estimated difference contributed by migraine or a MDE after controlling for five demographic variables. ${ }^{*} p<0.05 ;{ }^{* *} p<0.01$. 


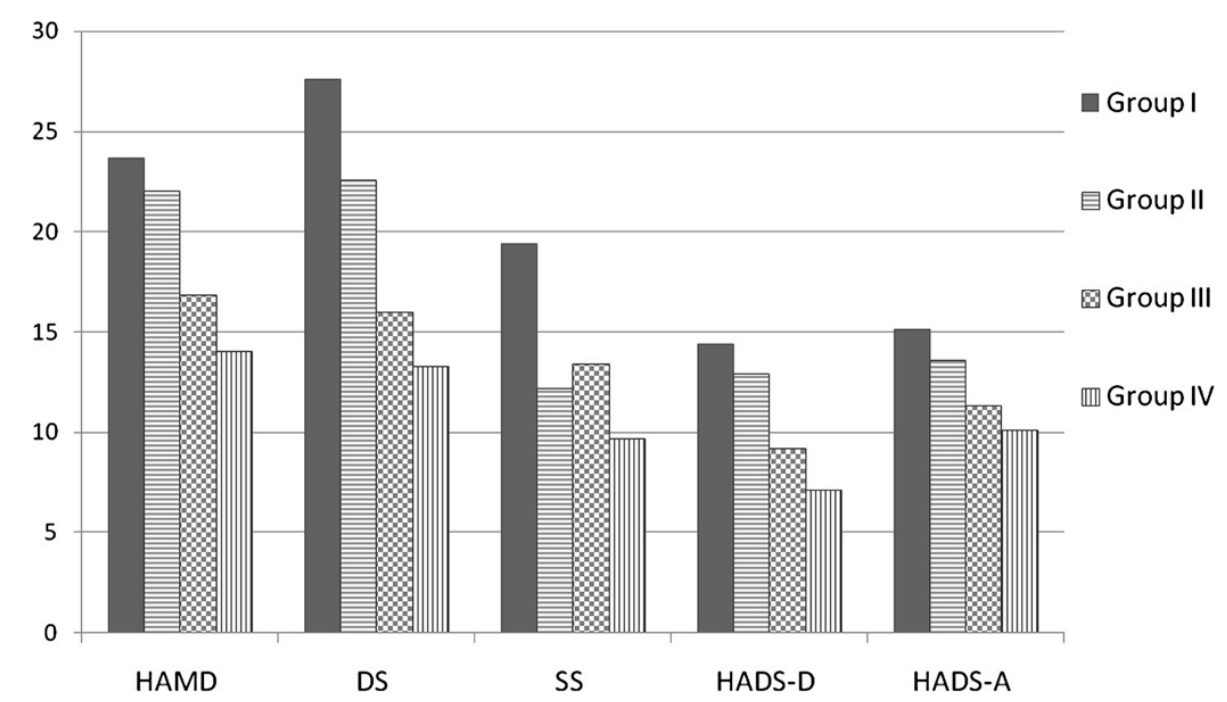

Figure 1 The severities of depression, anxiety, and somatic symptoms among patients with different diagnoses or comorbidities. Group I = a current major depressive episode (MDE) comorbid with migraine; Group II = a current MDE without migraine; Group III = migraine without a current MDE; Group IV = neither a current MDE nor migraine. HAMD Hamilton Depression Rating Scale, DS depression subscale of the Depression and Somatic Symptoms Scale (DSSS), SS somatic subscale of the DSSS, HADS-D depression subscale of the Hospital Anxiety and Depression Scale (HADS), HADS-A anxiety subscale of the HADS. Significance $(p<0.05)$ : Group I vs. II: DS and SS; Group I vs. III: All five subscales; Group I vs. IV: All five subscales; Group II vs. III: HAMD, DS, and HADS-D; Group II vs. IV: All five subscales except for SS; Group III vs. IV: none.

\section{Correlations of headache parameters to depression,} anxiety, somatic severity, and the SF-36

Headache intensity was significantly correlated to the three psychometric scales (the correlation coefficient $(r)$ ranged from $0.49 \mathrm{SS}$ to 0.29 HADS-D, all $p<0.01$ ) and the eight SF-36 subscales $(r: 0.49 \mathrm{BP}$ to $0.23 \mathrm{SF}$, all $p<0.01)$. The headache frequency was also significantly correlated to the three psychometric scales $(r: 0.54$ SS to 0.39 HADS-D, all $p<0.01)$ and all the SF-36 subscales ( $r$ : $0.47 \mathrm{BP}$ to $0.28 \mathrm{RP}$, all $p<0.05)$.

\section{The impacts of migraine on depression, anxiety, and} somatic symptoms

Table 5 identifies the factors that independently predict depression, anxiety, and somatic severities. After controlling for demographic variables and a current MDE, migraine was still an independent factor predicting the severities of depression, anxiety, and somatic symptoms. The variance of the SS explained by migraine was greater than that of a current MDE.

\section{The impacts of migraine on the SF-36 subscales}

After controlling for demographic variables and a current MDE, migraine was still an independent factor predicting all the SF-36 subscales, except for VT and $\mathrm{MH}$ (Table 5). The variance in the PF and the BP explained by migraine was greater than that of a current MDE.

\section{Discussion}

Our study showed that the presence of migraine among psychiatric outpatients was associated with a greater severity of depression, anxiety, and somatic symptoms, and a poorer HRQoL. Comorbidity with migraine explained a higher variance on the physical and pain symptoms compared to a current MDE. Depression and/or anxiety are often considered important factors related to the severity of somatic or pain symptoms; however, the role of migraine in somatic and pain symptoms in the psychiatric field has long been neglected. This study first pointed out that the importance of migraine was significantly greater than that of a MDE in predicting pain and somatic symptoms in the regression models.

Migraine might be a surrogate for somatic and pain symptoms among these patients. This is imperative because pain or somatic symptoms are common residual symptoms, which are related to a poorer prognosis [34], among depressive patients. It is possible that simultaneous prevention of migraine during treatment for mood and anxiety disorders might decrease residual somatic and pain symptoms and improve the prognosis. Therefore, physicians should integrate the treatment of migraine into psychiatric treatment plans. In fact, migraine was common (35.0\%) among these psychiatric outpatients. Patients with bipolar II disorder had the highest percentage (70\%) of migraine. This result is compatible with Fasmer's report (77\%) [19]. The percentage of migraine among patients with MDD was $49.1 \%$, which was close to that reported in 
Table 4 Comparison of the Short-Form 36 subscales and headache indices between outpatients with and without migraine and MDE

\begin{tabular}{|c|c|c|c|c|c|}
\hline & Total $(n=214)$ & & Yes & No & Estimated difference $^{\mathrm{a}}$ \\
\hline \multirow[t]{2}{*}{$\mathrm{PF}$} & $80.1 \pm 20.2$ & Migraine & $71.9 \pm 21.8^{* *}$ & $84.5 \pm 17.9$ & $-11.2^{* *}$ \\
\hline & & MDE & $74.4 \pm 20.9^{* *}$ & $84.7 \pm 18.5$ & $-9.4^{* *}$ \\
\hline \multirow[t]{2}{*}{$\mathrm{RP}$} & $39.7 \pm 40.2$ & Migraine & $23.7 \pm 36.0^{* *}$ & $48.4 \pm 39.7$ & $-23.7^{* *}$ \\
\hline & & MDE & $24.7 \pm 34.9^{* *}$ & $51.7 \pm 40.2$ & $-28.0^{* *}$ \\
\hline \multirow[t]{2}{*}{$\mathrm{BP}$} & $55.1 \pm 27.4$ & Migraine & $38.3 \pm 24.4^{* *}$ & $64.2 \pm 24.7$ & $-23.5^{* *}$ \\
\hline & & MDE & $43.6 \pm 25.6^{* *}$ & $64.4 \pm 25.4$ & $-20.0^{* *}$ \\
\hline \multirow[t]{2}{*}{$\mathrm{GH}$} & $39.3 \pm 21.0$ & Migraine & $31.3 \pm 21.5^{* *}$ & $43.6 \pm 19.5$ & $-11.6^{* *}$ \\
\hline & & MDE & $29.7 \pm 17.4^{* *}$ & $46.9 \pm 20.6$ & $-15.9^{* *}$ \\
\hline \multirow[t]{2}{*}{$V T$} & $31.1 \pm 22.0$ & Migraine & $21.6 \pm 17.1^{* *}$ & $36.3 \pm 22.7$ & $-13.2^{* *}$ \\
\hline & & MDE & $17.5 \pm 13.5^{* *}$ & $42.0 \pm 21.5$ & $-23.6^{* *}$ \\
\hline \multirow[t]{2}{*}{ SF } & $50.9 \pm 27.8$ & Migraine & $39.5 \pm 23.7^{* *}$ & $57.0 \pm 28.0$ & $-18.8^{* *}$ \\
\hline & & MDE & $36.6 \pm 23.2^{* *}$ & $62.3 \pm 25.9$ & $-25.7^{* *}$ \\
\hline \multirow[t]{2}{*}{ RE } & $26.5 \pm 37.0$ & Migraine & $11.6 \pm 27.7^{* *}$ & $34.5 \pm 39.0$ & $-22.8^{* *}$ \\
\hline & & MDE & $6.0 \pm 17.5^{* *}$ & $42.9 \pm 40.3$ & $-35.4^{* *}$ \\
\hline \multirow[t]{2}{*}{$\mathrm{MH}$} & $34.7 \pm 20.0$ & Migraine & $26.8 \pm 17.1^{* *}$ & $39.0 \pm 19.8$ & $-10.9^{* *}$ \\
\hline & & MDE & $22.7 \pm 12.0^{* *}$ & $44.3 \pm 19.4$ & $-20.5^{* *}$ \\
\hline \multirow[t]{2}{*}{$\mathrm{HI}$} & $3.3 \pm 3.4$ & Migraine & $6.0 \pm 3.3^{* *}$ & $1.9 \pm 2.5$ & \\
\hline & & MDE & $4.7 \pm 3.4^{* *}$ & $2.2 \pm 3.0$ & \\
\hline \multirow[t]{2}{*}{$\mathrm{HF}$} & $2.6 \pm 2.8$ & Migraine & $4.6 \pm 2.6^{* *}$ & $1.6 \pm 2.2$ & \\
\hline & & $\mathrm{MDE}$ & $3.9 \pm 2.8^{* *}$ & $1.6 \pm 2.2$ & \\
\hline
\end{tabular}

PF physical functioning, RP role limitations-physical, BP bodily pain, GH general health perceptions, VT vitality, SF social functioning, RE role limitations-emotional, $\mathrm{MH}$ mental health, $\mathrm{HI}$ average headache intensity in the past week, HF headache days in the past week, MDE a current major depressive episode.

${ }^{a}$ Estimated difference contributed by migraine or a MDE after controlling for five demographic variables. ${ }^{* *} p<0.006$.

previous studies $[14,19]$. The percentage of migraine among patients with only anxiety disorder was $24.5 \%$, which was lower than that reported in the study of Senaratne et al. (67\%) in an anxiety disorders clinic [17]. This might partially result from our diagnostic algorithm, i.e., in patients with only anxiety disorder, other psychiatric comorbidities, such as MDD and bipolar disorders, were excluded.

Our results that psychiatric outpatients with migraine were associated with more somatic or pain symptoms might be explained by two possible reasons. 1) Imbalance of neurotransmitters, such as serotonin, noradrenalin, and dopamine, is responsible for the pathogenesis of migraine as well as somatic symptoms and depression [35,36]. This is commonly used to explain the close relationship between migraine, depression, and somatic symptoms; 2) Repeated headache attacks might cause a similar effect to central sensitization, which is associated with comorbid pain conditions and a worse headache-related disability and increases somatic or pain symptoms among psychiatric outpatients with migraine [37].

There are several points, which might be helpful for clinical practice, worth noting. 1) Headache intensity and frequency were significantly correlated to the severity of depression, anxiety, somatic symptoms, and HRQoL. Therefore, headache intensity and frequency could be a useful index to predict these mental symptoms and HRQoL. 2) Depression and migraine independently predicted negative impacts on quality of life in a general population [38]. Our results demonstrated that migraine and a current MDE were independently associated with depression, anxiety, somatic symptoms, and HRQoL among these psychiatric outpatients. 3) The study showed that patients with both a MDE and migraine had the greatest severity of depression, anxiety, and somatic symptoms. This demonstrated the synergic effect of depression and migraine. 4) Psychiatric outpatients with migraine had an increased risk of a suicide attempt history. This result was compatible with previous studies [39]. In fact, migraine has been neglected in terms of evaluating suicide risk among psychiatric outpatients.

Our study has the following limitations. 1) Our study enrolled psychiatric outpatients in a medical center with several exclusion criteria to prevent confounding effects. This enrollment process might introduce bias. For example, the study excluded patients with a current manic episode or with psychotic features. Therefore, this study did not enroll patients with bipolar I disorder. 2) The 
Table 5 Independent factors predicting depression, anxiety, somatic symptoms, and health-related quality of life among psychiatric outpatients

\begin{tabular}{|c|c|c|c|c|}
\hline & Independent variable & Beta & $R^{2}$ change & $p$ value \\
\hline \multirow[t]{4}{*}{ HAMD } & MDE & 0.58 & 0.44 & $<0.01$ \\
\hline & Being employed & -0.15 & 0.03 & $<0.01$ \\
\hline & Migraine & 0.15 & 0.02 & $<0.01$ \\
\hline & Age & -0.11 & 0.01 & 0.03 \\
\hline \multirow[t]{4}{*}{ DS } & MDE & 0.57 & 0.45 & $<0.01$ \\
\hline & Migraine & 0.20 & 0.04 & $<0.01$ \\
\hline & Age & -0.12 & 0.01 & 0.01 \\
\hline & Gender & -0.11 & 0.01 & 0.03 \\
\hline \multirow[t]{4}{*}{ SS } & Migraine & 0.32 & 0.23 & $<0.01$ \\
\hline & MDE & 0.27 & 0.06 & $<0.01$ \\
\hline & Being employed & -0.15 & 0.04 & 0.01 \\
\hline & Gender & -0.15 & 0.02 & 0.02 \\
\hline \multirow[t]{3}{*}{ HADS-D } & MDE & 0.57 & 0.39 & $<0.01$ \\
\hline & Migraine & 0.16 & 0.03 & 0.01 \\
\hline & Being employed & -0.12 & 0.01 & 0.03 \\
\hline \multirow[t]{3}{*}{ HADS-A } & MDE & 0.41 & 0.21 & $<0.01$ \\
\hline & Being employed & -0.13 & 0.02 & 0.03 \\
\hline & Migraine & 0.14 & 0.02 & 0.04 \\
\hline \multirow[t]{3}{*}{ PF } & Migraine & -0.22 & 0.09 & $<0.01$ \\
\hline & MDE & -0.17 & 0.02 & 0.02 \\
\hline & Being employed & 0.14 & 0.02 & 0.03 \\
\hline \multirow[t]{2}{*}{ RP } & MDE & -0.26 & 0.11 & $<0.01$ \\
\hline & Migraine & -0.20 & 0.03 & $<0.01$ \\
\hline \multirow[t]{3}{*}{ BP } & Migraine & -0.35 & 0.20 & $<0.01$ \\
\hline & MDE & -0.25 & 0.05 & $<0.01$ \\
\hline & Being employed & 0.13 & 0.02 & 0.03 \\
\hline \multirow[t]{3}{*}{ GH } & MDE & -0.36 & 0.17 & $<0.01$ \\
\hline & Being employed & 0.15 & 0.03 & 0.01 \\
\hline & Migraine & -0.13 & 0.02 & 0.045 \\
\hline \multirow[t]{2}{*}{ VT } & MDE & -0.55 & 0.31 & $<0.01$ \\
\hline & Being employed & 0.17 & 0.03 & $<0.01$ \\
\hline \multirow[t]{3}{*}{ SF } & MDE & -0.40 & 0.21 & $<0.01$ \\
\hline & Migraine & -0.17 & 0.02 & 0.01 \\
\hline & Educational years & -0.15 & 0.02 & 0.01 \\
\hline \multirow[t]{4}{*}{ RE } & MDE & -0.42 & 0.25 & $<0.01$ \\
\hline & Age & 0.15 & 0.02 & 0.01 \\
\hline & Being employed & 0.12 & 0.02 & 0.04 \\
\hline & Migraine & -0.13 & 0.01 & 0.04 \\
\hline $\mathrm{MH}$ & MDE & -0.52 & 0.30 & $<0.01$ \\
\hline
\end{tabular}

Table 5 Independent factors predicting depression, anxiety, somatic symptoms, and health-related quality of life among psychiatric outpatients (Continued)

\begin{tabular}{|c|c|c|c|}
\hline Being employed & 0.16 & 0.03 & $<0.01$ \\
\hline Age & 0.12 & 0.01 & 0.04 \\
\hline \multicolumn{4}{|c|}{$\begin{array}{l}\text { HAMD Hamilton Depression Rating Scale, DS depression subscale of the } \\
\text { Depression and Somatic Symptoms Scale (DSSS), SS somatic subscale of the } \\
\text { DSSS, HADS-D depression subscale of the Hospital Anxiety and Depression } \\
\text { Scale (HADS), HADS-A anxiety subscale of the HADS, PF physical functioning, } \\
\text { RP role limitations-physical, BP bodily pain, GH general health perceptions, VT } \\
\text { vitality, SF social functioning, RE role limitations-emotional, MH mental health, } \\
\text { MDE a current major depressive episode. }\end{array}$} \\
\hline
\end{tabular}

sample size of some diagnoses, such as bipolar II disorder, was small. The study design, mixing different diagnoses of mood and anxiety disorders, had the advantage of reflecting the percentage of migraine among global nonpsychotic outpatients. 3) The headache intensity and frequency in the past week were recalled by patients. Collecting data on the two headache parameters prospectively by headache diary might be more reliable. However, withholding pharmacological treatment while prospectively observing headache parameters might result in ethical problems owing to the suicidal risk. 4) Depression and migraine are associated with an increased suicide risk. In the study, only the past history of suicide attempts was recorded. A comprehensive assessment of suicide risk was not performed.

\section{Conclusions}

In conclusion, migraine was common among psychiatric outpatients with mood and/or anxiety disorders. Bipolar II disorder patients had the highest percentage of migraine, followed by MDD. Patients with migraine had a greater severity of depression, anxiety, and somatic symptoms, as well as a poorer HRQoL than those without. Of note, the association of migraine with $\mathrm{PF}, \mathrm{BP}$, and somatic symptoms might be greater than that of a current MDE. Because of the high frequency and significant impacts of migraine on physical and pain symptoms, future studies should explore how to integrate the treatment strategies of migraine, mood disorders and anxiety disorders.

Competing interests

All authors declare that they have no competing interests.

Authors' contributions

SJW and $\mathrm{ClH}$ designed the study and wrote the protocol. $\mathrm{ClH}$ and $\mathrm{CYL}$ collected the data. SJW and $\mathrm{CIH}$ undertook the statistical analysis, and $\mathrm{CIH}$ wrote the first draft of the manuscript. All authors read and approved the final manuscript.

\section{Acknowledgements}

This study was supported in part by grants from the National Science Council of Taiwan (NSC 95-2314-B-182A-188-MY2), Taipei Veterans General Hospital [VGHUST101-G7-1-1, V101C-106, V101E7-003], NSC support for Centre for Dynamical Biomarkers and Translational Medicine, National Central University, Taiwan [NSC100-2911-I-008-001], Brain Research Center, National 
Yang-Ming University, and a grant from Ministry of Education, Aim for the Top University Plan.

\section{Author details}

'Department of Psychiatry, Chang-Gung Memorial Hospital at Linkou and Chang-Gung University School of Medicine, Taoyuan, Taiwan. ${ }^{2}$ Neurological Institute, Taipei Veterans General Hospital and National Yang-Ming University School of Medicine, No. 201 Shi-Pai Road, Section 2, Taipei 112, Taiwan.

\section{Received: 16 November 2012 Accepted: 19 February 2013}

Published: 27 February 2013

\section{References}

1. Jette N, Patten S, Williams J et al (2008) Comorbidity of migraine and psychiatric disorders-a national population-based study. Headache 48:501-516

2. Cripe SM, Sanchez S, Lam N et al (2010) Depressive symptoms and migraine comorbidity among pregnant Peruvian women. J Affect Disord 122:149-153

3. Victor TW, Hu X, Campbell J et al (2010) Association between migraine, anxiety and depression. Cephalalgia 30:567-575

4. Baskin SM, Smitherman TA (2009) Migraine and psychiatric disorders: comorbidities, mechanisms, and clinical applications. Neurol Sci 30(Suppl 1): S61-S65

5. Ratcliffe GE, Enns MW, Jacobi F et al (2009) The relationship between migraine and mental disorders in a population-based sample. Gen Hosp Psychiatry 31:14-19

6. Samaan Z, Farmer A, Craddock N et al (2009) Migraine in recurrent depression: case-control study. Brit J Psychiatry 194:350-354

7. Beghi E, Bussone G, D'Amico D et al (2010) Headache, anxiety and depressive disorders: the HADAS study. J Headache Pain 11:141-150

8. Radat F, Mekies C, Geraud G et al (2008) Anxiety, stress and coping behaviours in primary care migraine patients: results of the SMILE study. Cephalalgia 28:1115-1125

9. Smitherman TA, Penzien DB, Maizels M (2008) Anxiety disorders and migraine intractability and progression. Curr Pain Headache Rep 12:224-229

10. Heckman BD, Holroyd KA, Himawan L et al (2009) Do psychiatric comorbidities influence headache treatment outcomes? Results of a naturalistic longitudinal treatment study. Pain 146:56-64

11. Pompili M, Di Cosimo D, Innamorati M et al (2009) Psychiatric comorbidity in patients with chronic daily headache and migraine: a selective overview including personality traits and suicide risk. J Headache Pain 10:283-290

12. Ligthart L, Penninx BW, Nyholt DR et al (2010) Migraine symptomatology and major depressive disorder. Cephalalgia 30:1073-1081

13. Hung Cl, Liu CY, Cheng TY et al (2009) Migraine: A missing link between somatic symptoms and major depressive disorder. J Affect Disord 117:108-115

14. Hung Cl, Liu CY, Juang YY et al (2006) The impact of migraine on patients with major depressive disorder. Headache 46:469-477

15. Hung Cl, Wang SJ, Yang CH et al (2008) The impacts of migraine, anxiety disorders, and chronic depression on quality of life in psychiatric outpatients with major depressive disorder. J Psychosom Res 65:135-142

16. Oedegaard KJ, Fasmer OB (2005) Is migraine in unipolar depressed patients a bipolar spectrum trait? J Affect Disord 84:233-242

17. Senaratne $R$, Van Ameringen M, Mancini C et al (2010) The prevalence of migraine headaches in an anxiety disorders clinic sample. CNS Neurosci Ther 16:76-82

18. Peterlin BL, Rosso AL, Sheftell FD et al (2011) Post-traumatic stress disorder, drug abuse and migraine: new findings from the National Comorbidity Survey Replication (NCS-R). Cephalalgia 31:235-244

19. Fasmer $O B$ (2001) The prevalence of migraine in patients with bipolar and unipolar affective disorders. Cephalalgia 21:894-899

20. McIntyre RS, Konarski JZ, Wilkins K et al (2006) The prevalence and impact of migraine headache in bipolar disorder: results from the Canadian Community Health Survey. Headache 46:973-982

21. Mclntyre RS, Liauw S, Taylor VH (2011) Depression in the workforce: the intermediary effect of medical comorbidity. J Affect Disord 128(Suppl 1): S29-S36

22. Blumenfeld AM, Varon SF, Wilcox TK et al (2011) Disability, HRQoL and resource use among chronic and episodic migraineurs: Results from the International Burden of Migraine Study (IBMS). Cephalalgia 31:301-315

23. Cheng TM (2003) Taiwan's new national health insurance program: genesis and experience so far. Health Aff 22:61-76
24. Wu CH, Kao JC, Chang CJ (1996) Analysis of outpatient referral failures. J Fam Pract 42:498-502

25. Hung Cl, Wang SJ, Liu CY (2009) Validation of the Depression and Somatic Symptoms Scale (DSSS) by comparison with the Short Form 36 (SF-36) scale among psychiatric outpatients with major depressive disorder. Depress Anxiety 26:583-591

26. Hung Cl, Liu CY, Wang SJ et al (2010) Somatic symptoms: An important index in predicting the outcome of depression at six-month and two-year follow-up points among outpatients with major depressive disorder. J Affect Disord 125:134-140

27. Hung Cl, Liu CY, Wang SJ et al (2012) The cut-off points of the Depression and Somatic Symptoms Scale and the Hospital Anxiety and Depression Scale in detecting non-full remission and a current major depressive episode. Int J Psychiatry Clin Pract 16:33-40

28. Hamilton M (1967) Development of a rating scale for primary depressive illness. Br J Soc Clin Psychol 6:278-296

29. Zigmond AS, Snaith RP (1983) The Hospital Anxiety and Depression Scale. Acta Psychiat Scand 67:361-370

30. Ware JE, Sherbourne CD (1992) The MOS 36-item short-form health survey (SF-36). I. Conceptual framework and item selection. Med Care 30:473-483

31. Tseng HM, Lu JF, Tsai YJ (2003) Assessment of health-related quality of life, II: Norming and validation of SF-36 Taiwan Version. Taiwan J Public Health 22:512-518

32. First MB, Spitzer RL, Gibbon M, Williams JBW (2002) Structured Clinical Interview for DSM-IV-TR Axis I Disorders, Research Version, Patient Edition (SCID-I/P). Biometrics Research, New York State Psychiatric Institute, New York

33. Headache Classification Subcommittee of the International Headache Society (2004) The International Classification of Headache Disorders, 2nd ed. Cephalalgia 24:1-160

34. Paykel ES (2008) Partial remission, residual symptoms, and relapse in depression. Dialogues Clin Neurosci 10:431-437

35. D'Andrea G, Leon A (2010) Pathogenesis of migraine: from neurotransmitters to neuromodulators and beyond. Neurol Sci 31:1-7

36. Kapfhammer HP (2006) Somatic symptoms in depression. Dialogues Clin Neurosci 8:227-239

37. Tietjen GE, Brandes JL, Peterlin BL et al (2009) Allodynia in migraine: association with comorbid pain conditions. Headache 49:1333-1344

38. Lipton RB, Hamelsky SW, Kolodner KB et al (2000) Migraine, quality of life, and depression: a population-based case-control study. Neurology 55:629-635

39. Wang SJ (2007) Migraine and suicide. Expert Rev Neurother 7:1069-1071

\section{doi:10.1186/1129-2377-14-19}

Cite this article as: Hung et al: Migraine predicts physical and pain symptoms among psychiatric outpatients. The Journal of Headache and Pain 2013 14:19.

\section{Submit your manuscript to a SpringerOpen ${ }^{\odot}$ journal and benefit from:}

- Convenient online submission

$\checkmark$ Rigorous peer review

- Immediate publication on acceptance

- Open access: articles freely available online

- High visibility within the field

- Retaining the copyright to your article

Submit your next manuscript at springeropen.com 